\title{
Conducting ballistic magnetoresistance and tunneling magnetoresistance: Pinholes and tunnel barriers
}

\author{
N. García ${ }^{a}$ \\ Los Mochuelos, Calapanizo, Cuevas del Almazora, Almería, Spain and Laboratorio de Física \\ de Sistemas Pequeños y Nanotecnología, Consejo Superior de Investigaciones Científicas (CSIC), \\ Serrano 144, E-28006 Madrid, Spain
}

(Received 2 February 2000; accepted for publication 10 July 2000)

\begin{abstract}
This letter shows that conducting ballistic and tunneling magnetoresistances have identical physical treatments and, therefore, it is not possible to distinguish whether an experiment is measuring, in oxide barriers, electron conduction through pinholes or tunneling because both types of conduction are governed by the same physics. Currents in both cases are calculated by matching wave functions at the electrodes (both are ballistic). Therefore, pinholes or small ballistic nanocontacts in the tunnel barrier allow conducting ballistic electrons that account for most of the current. We also show that, in the case of nonsuperconducting materials, e.g., magnetic materials, current-voltage characteristics are not suitable to distinguish one process from the other. (c) 2000 American Institute of Physics. [S0003-6951(00)04835-X]
\end{abstract}

Experiments in ferromagnetic $(F) /$ oxide $(O) /$ superconductor $(S)$ junctions were done to determine the spin polarization of the electron tunneling from $F$ to $S$ by using the properties of the $S$ gap. ${ }^{1}$ In all the experiments performed with $3 d$ metals, the electron polarization at the Fermi level $\left(E_{f}\right)$ was found to be positive; however, band-structure calculations $^{2}$ had shown that the polarization at $E_{f}$ of $3 d$ metals ( $\mathrm{Ni}$ and $\mathrm{Co}$ ) is negative due to the large density of $d$ minority electrons; i.e., the minority electrons at $E_{f}$ have a larger density of states than the majority electrons. The fact that the polarization observed was positive for all $d$ metals was interpreted as indicative that, even when the bulk material is negatively polarized, the tunnel electrons are $s$ electrons, being positively polarized at $E_{f}{ }^{1,3,4}$ On the other hand, recent experiments had shown very large values of ballistic magnetoresistance (BMR), up to $300 \%$, in nanocontacts bridging two $3 d$ ferromagnets $(\mathrm{Ni}$ and $\mathrm{Co}$ ) at room temperature (RT) and for a maximum applied magnetic field of 120 Oe. ${ }^{5}$ Furthermore, the theory to understand these experiments shows ${ }^{5}$ that in this case the electrons were negatively polarized; i.e., the experimental results are explained by considering an overwhelming large density of states of $d$ electrons with respect to $s$ electrons at $E_{f}$.

The theory for BMR (Ref. 5) shows that for the case of identical ferromagnets at both sides of the nanocontact,

$$
\mathrm{BMR}=\frac{R_{a p}-R_{p}}{R_{p}}=\frac{2 P^{2}}{1-P^{2}} \times F\left(\lambda, k_{f}\right),
$$

where $R_{a p}$ and $R_{p}$ are the resistance for the antiferromagnetic and ferromagnetic configurations. $P=\left(D_{\uparrow}-D_{\downarrow}\right) /\left(D_{\uparrow}\right.$ $\left.+D_{\downarrow}\right)$ is the electron polarization assuming that all the electrons $s$ and $d$ have the same ballistic transmitivity, approximately unity, through the contact; $\lambda$ and $k_{f}$ are the domainwall width and the Fermi vector, respectively, and $F$ is a function describing the domain-wall scattering or nonspin conservation in the current. If $\lambda$ at the nanocontact is very

${ }^{a}$ Electronic mail: nicolas.garcia@fsp.csic.es small (a few atomic layers) then we are in the nonadiabatic limit $F=1$, spins cannot flip by domain-wall collision and spin conservation occurs. Then, we obtain very large magnetoresistance (MR) if the polarization prefactor is large, ${ }^{5}$

$$
\mathrm{BMR}=\frac{2 P^{2}}{1-P^{2}} .
$$

This is the same expression that has been deduced for explaining tunneling magnetoresistance, ${ }^{6}$ or one can also use $\left(R_{a p}-R_{p}\right) / R_{a p}=2 P^{2} /\left(1+P^{2}\right)$ Julliere's formula. ${ }^{6,7}$ Identical expressions are obtained for conductive ballistic magnetoresistance and tunneling magnetoresistance (TMR) if it is assumed that all electrons ( $s$ and $d$ ) have the same tunneling probability. ${ }^{6,7}$ This is not so because $s$ electrons have larger tunnel transmitivities than $d$ electrons. From theory, $P$ can be generalized to $\mathrm{be}^{4}$

$$
P_{g}=\frac{\left(D_{s \uparrow}-D_{s \downarrow}\right) t_{s}+\left(D_{d \uparrow}-D_{d \downarrow}\right) t_{d}}{\left(D_{s \uparrow}+D_{s \downarrow}\right) t_{s}+\left(D_{d \uparrow}+D_{d \downarrow}\right) t_{d}}
$$

where $\left(D_{s \uparrow}, D_{s \downarrow}\right)$ and $\left(t_{s}, t_{d}\right)$ are density of states and transmitivities for $s$ and $d$ electrons up and down at $E_{f}$. By inserting Expression (3) into (1), both tunneling and conducting experimental results can be explained. Notice that $t_{s}$ $\sim e^{-\phi^{1 / 2} l}$ and $t_{d} \sim e^{-\left(\phi+E_{L}\right)^{1 / 2} l}$, where $E_{L}$ and $l$ are the angular momentum energy and barrier width. ${ }^{4}$ Also, for $3 d$ metals, band-structure calculations show that $D_{s \uparrow}>D_{s \downarrow}$ and $D_{d \downarrow}>D_{d \uparrow}$. Therefore, for tunneling, the $s$ electrons dominate and the polarization could be positive for $l \geqslant 0.4 \mathrm{~nm}$ and for $D_{s \uparrow}>D_{s \downarrow},{ }^{2,3}$ which is in agreement with experiments. ${ }^{1}$ However, in conducting ballistic BMR experiments with $\mathrm{Ni}$ and Co, $t_{s} \sim t_{d} \sim 1, D_{d \downarrow} \gg D_{d \uparrow}, D_{s \uparrow}, D_{s \downarrow},{ }^{2}$ and the polarization is negative as shown by the BMR experiments. ${ }^{5}$

If the two electrodes are different ferromagnets $\mathrm{F} 1$ and F2, the prefactor in formula (1) becomes $2 P_{g_{1}} P_{g_{2}} /(1$ $\left.-P_{g 1} P_{g 2}\right)$, where now $P_{g 1}$ and $P_{g 2}$ are the polarizations for F1 and F2 according to formula (3).

Why is the same formula valid for BMR and TMR? The answer is that both processes are due to the same physical 
principles and are obtained by matching the wave functions at both sides of the contact (or of the tunnel barrier, which for the case, is the same). The only difference is in the transmitivities; for conducting ballistic electrons the transmitivity values are of the order of unity, while for tunneling the transmitivities decay exponentially with $l$. But, this brings up the question: how do we know in an experiment if we have tunneling or conducting electrons? There is no way to know and this cannot be known because both processes are ballistic. ${ }^{4,8,9}$ In experiments with superconductors, observations of superconducting gaps, phonon excitations, Andreev reflection, etc., are common to both conducting ballistic and tunneling electrons. The distinction between ballistic and tunneling currents may be obtained by comparing in detail the dependence of conductances with $T$ and $V$, and then differences in the behavior of the currents appear because of the presence of the superconductor gap. However, in conductances between two magnetic electrodes the subtleties existing in superconductors disappear and conducting ballistic or tunneling currents cannot be distinguished and, therefore, from MR measurements alone it is not possible to distinguish if the currents result from tunneling or from pinholes. Notice that a pinhole of $\sim 1 \mathrm{~nm}$ size contributes as much as a $\sim 1$ $\mu$ m-size tunnel barrier $\left(1 \mathrm{~nm}\right.$ width of $\left.\mathrm{Al}_{2} \mathrm{O}_{3}\right)$.

It has been proposed that conduction from pinholes and tunneling in oxide barriers can be distinguished from current-voltage $(I-V)$ characteristics. ${ }^{10}$ The argument is that the $I-V$ are linear for conduction through pinholes and nonlinear for tunneling. However, it should be mentioned that $I-V$ tunneling characteristics between metallic electrodes at low $T$ and $V$ should also be linear. This is what one obtains from tunneling theory, but not otherwise because $I$ $\propto V \times \exp (-\sqrt{\phi} l) .{ }^{4}$ We suggest that the nonlinear characteristics in Ref. 10 may arise as a consequence of a semiconducting state formed at the ferromagnet-oxide interfaces. The curves of Figs. 1 and 2 in Ref. 10 are typical for semiconducting electrodes. Also, the $I-V$ characteristics for small nanocontacts or pinholes are nonlinear. In Fig. 1, $I-V$ curves for nanocontacts in Ni show that for very small nanocotacts $\left(n=1-7\right.$ quanta of conductance $\left.2 e^{2} / h\right)$, the $I-V$ are nonlinear and become linear when the nanocontacts are larger. This may be due to some electron-electron interaction that is important for small $n$. It should be mentioned that when $n=1-3$ we have measured BMR up to $300 \%$. Similar $I-V$ curves were measured for Au nanocontacts. ${ }^{11}$ Then, the $I-V$ characteristics are not valid criteria to indicate pinhole or tunnel conductance. The only way to distinguish pinholes from tunnel barrier currents is by measuring the dependence of the current with the insulator thickness. In the case of tunneling, the dependence is exponential; however, in magnetic multilayers this cannot be done. Only local probes, such as scanning tunneling microscopy, can see those effects that are local, i.e., in some points of the film, the conduction is by tunneling, and in others, by pinholes.

There is a point that should be stressed. For thick oxides it is possible that the pinholes in the barrier are all oxidized, and also, that long pinholes larger than, say, $3-5 \mathrm{~nm}$ may be nonballistic. However, in the case of thin oxides with resistance smaller than $10^{4} \Omega$ or barrier thickness smaller than 1 or $2 \mathrm{~nm}$, it is hazardous to claim only tunneling and no Downloaded 10 Feb 2010 to 161.111 .180 .191 . Redistribution subje

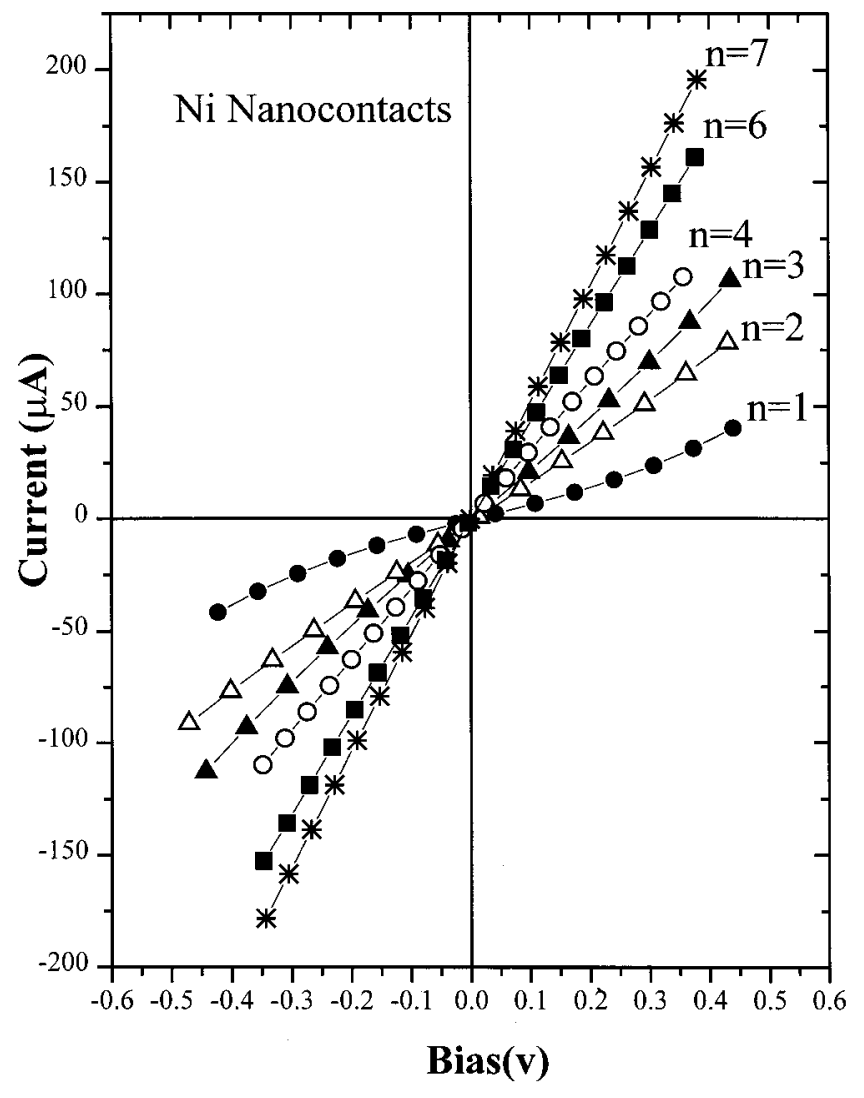

FIG. 1. $I-V$ experimental curves for ballistic Ni nanocontacts for the quanta of conductance $n=1-7$. Notice the nonlinearity for small $n$. For $n=7$, the curves are linear up to $0.5 \mathrm{~V}$ bias. For $n<3$, values of BMR up to $300 \%$ were observed (see Ref. 5).

conducting ballistic pinholes or nanocontacts currents, and it will not be surprising that the MR contributions are dominated by pinholes because in this case $d$ electrons will contribute and these are the ones that produce large MR values because of their high density of states at $E_{f}$.

We can estimate the values of $\phi$, the tunnel barrier height, from the experimental measurements of resistance. If there is no tunnel barrier, $\phi<0$, then it is conducting ballistic, $T \approx 1$, the resistance of one atom is approximately $10^{4} \Omega$, the quantum of resistance. Therefore, for $1 \mu \mathrm{m}^{2}$, the resistance is $\sim 10^{-3} \Omega\left(1 \mu \mathrm{m}^{2} \sim 10^{7}\right.$ surface atoms $)$. When the tunnel barrier is present, the resistance for the $1 \mu \mathrm{m}^{2}$ area is $R_{T}\left(1 \mu \mathrm{m}^{2}\right) \approx\left(10^{-3} / T_{T}\right) \Omega$, where $T_{T}$ is the tunneling transmitivity, and then $\phi$ (in $\mathrm{eV}$ ) is

$$
\phi^{1 / 2}=-\frac{1}{10 l} \log \left(\frac{10^{-3}}{R_{T}}\right),
$$

with the barrier thickness $l$ in nanometers. For resistances of 200 and $10^{6} \Omega$ in a $100 \mu \mathrm{m}^{2}$ area and $l \approx 2.5 \mathrm{~nm}$, we have $\phi \approx 0.45$ and $\sim 1.03 \mathrm{eV}$, respectively. It is hard to believe that with a $0.45 \mathrm{eV}$ mean tunneling barrier in $100 \mu \mathrm{m}^{2}$, there are no pinholes.

Another point is that we have discussed the case in which $F=1$ in formula (1) (the case of spin conservation); i.e., when $k_{f} \lambda$ tends to zero. However, this is not necessarily true and, in fact, we have shown ${ }^{5}$ that for BMR the product $k_{f} \lambda$ grows with the contact size and $F$ decreases very fast, which reduces the BMR effect (nonconservation of the spin). A similar behavior will occur in tunneling. In fact, it will be to AIP license or copyright; see http://apl.aip.org/apl/copyright.jsp 
more pronounced because of a larger interface area. Therefore, the TMR is not given only by the polarization prefactor in formula (1), but one has to take into account the function $F$ as well. This will be discussed in detail in a forthcoming paper which will consider the influence of the interface magnetic properties through the function $F$. Therefore, MR can be small even if the polarization is large due to nonconservation of spin by scattering with domain walls. ${ }^{5}$ In fact, this is what happens in bulk ferromagnets due to the large domain-wall width.

This work has been supported by the Spanish DGICyT. The author thanks his students Y.-W. Zhao and M. Muñoz for producing the curves of Fig. 1.

${ }^{1}$ R. Meservey and P. M. Tedrow, Phys. Rep. 238, 173 (1994), and references therein.

${ }^{2}$ J. L. Moruzzi, J. F. Janak, and A. R. Williams, Calculated Band Structure Properties (Pergamon, New York, 1978).

${ }^{3}$ M. B. Stearns, J. Magn. Magn. Mater. 5, 167 (1977).
${ }^{4}$ N. García (unpublished); the tunnel transmitivities are larger for $s$ than for $d$ electrons because the kinetic energy of $d$ electrons has a large component of rotational energy $E_{L}$ due to their angular momentum $L=2$, while for $s$ electrons $L=0$. Therefore, the tunnel barriers are $\phi$ and $\phi+E_{L}$ for $s$ and $d$ electrons, respectively; F. Guinea, M. J. Calderon, and L. Brey (unpublished).

${ }^{5}$ N. García, M. Muñoz, and Y.-W. Zhao, Phys. Rev. Lett. 82, 2923 (1999); N. García, M. Muñoz, and Y.-W. Zhao, Appl. Phys. Lett. 76, 2586 (2000). G. Tatatra, Y.-W. Zhao, M. Muñoz, and N. Garcia, Phys. Rev. Lett. 83, 2030 (1999).

${ }^{6}$ J. S. Moodera and G. Mathon, J. Magn. Magn. Mater. 200, 248 (1999), and references therein.

${ }^{7}$ M. Julliere, Phys. Lett. 54A, 225 (1975).

${ }^{8}$ N. García, H. Rohrer, M. Muñoz, and Y.-W. Zhao, Spanish Patent No. P9802091 (1998), International PCT application.

${ }^{9}$ C. L. Platt, A. S. Katz, R. C. Dynes, and A. E. Berkowitz, Appl. Phys. Lett. 75, 127 (1999).

${ }^{10}$ C. L. Platt, B. Dieny, and A. E. Berkowitz, J. Appl. Phys. 81, 5523 (1997).

${ }^{11}$ J. L. Costa-Krämer, N. García, P. García-Mochales, P. A. Serena, M. I. Marques, and A. Correia, Phys. Rev. B 55, 5416 (1997); N. García and J. L. Costa-Krämer, Europhys. News 27, 89 (1996); J. L. Costa-Krämer, N. García, M. Jonson, R. V. Kriye, H. Olin, P. A. Serena, and R. I. Shekhter, NATO ASI Ser., Ser. E 348, 1 (1998). 